\title{
Runtime and Space Complexity Comparison of the various Association Algorithms
}

\author{
K. Fathima Bibi, \\ Research Scholar, \\ Reg. No.:Ph.D_CB_Dec2012_0441, \\ Dept. of Comp. Sc., Bharathiyar University, \\ Coimbatore, TN, India
}

\author{
M. Nazreen Banu, Ph.D \\ Research Advisor, \\ Professor, Dept. of Comp. Sc. \& Engg, \\ MAM College of Engineering, \\ Tiruchirappalli, TN, India
}

\begin{abstract}
Data mining has become an indispensable technology for businesses and researchers in many fields. Discovering frequent itemsets is a key problem in important data mining applications. Typical association algorithms for solving this problem operate in a bottom-up, top-down and breadth-first search direction. The computation starts from frequent 1-itemsets (the minimum length frequent itemsets) and continues until all maximal (length) frequent itemsets are found. Algorithms perform well when all maximal frequent itemsets are short. However, performance drastically decreases when some of the maximal frequent itemsets are relatively long. This paper focuses on finding Maximum Frequent Set with the implementation of the APRIORI and the Dynamic Itemset Counting Algorithm (DIC) and a comparative study with Pincer Search Algorithm to select the fast algorithm for discovering the Maximum Frequent Set.
\end{abstract}

\section{General Terms}

Knowledge Discovery, Data Warehousing, Data Mining, Algorithms, Patterns.

\section{Keywords}

Maximum Frequent Set, Association, Classification, Clustering, Sequential, Outlier, Evolution.

\section{INTRODUCTION}

Data Warehousing: The database in a data warehouse is not the same as the databases used for transaction processing. Data warehouse databases are designed to analyze terabytes of data and millions of records. They are organized to allow better analysis by using special techniques.

\section{Data Mining:}

- hot buzzword for a class of techniques that find patterns in data [1]

- A user-centric, interactive process which leverages analysis technologies and computing power

- A group of techniques that find relationships that have not previously been discovered

Knowledge Discovery in Database (KDD) defined as "the non-trivial process of identifying valid, novel, potentially useful, and ultimately understandable patterns in data". A key component of many data mining problems is formulated as follows. Given a large database of sets of items, discover all frequent itemsets (sets of items), where a frequent itemset is one that occurs in at least a userdefined percentage (minimum support) of the database.

The problem was first formulated by Agrawal et al. [2, 3, $4,5,6,7,8,9]$ and is often referred to as the "marketbasket" problem. In this problem, a set of items and a large collection of transactions which are subsets (baskets) of these items are given. The task is to find relationship between the presences of various items within those baskets.

\section{METHODOLOGIES}

Different methods or techniques are proposed based on which the hidden patterns are discovered. They are Association Analysis, Classification, Prediction, Cluster Analysis, Sequential Pattern Analysis, Outlier Analysis and Evolution Analysis.

\subsection{Association Analysis}

As defined by Agrawal et al.,

Let $I=\left\{i_{1}, i_{2}, \ldots \ldots, i_{n}\right\}$ be a set of $n$ binary attributes called items.

Let $\mathrm{D}=\left\{\mathrm{t}_{1}, \mathrm{t}_{2}, \ldots \ldots, \mathrm{t}_{\mathrm{m}}\right\}$ be a set of transactions called the database.

Each transaction in D has a unique transaction ID and contains a subset of the items in I.

An Association rule is defined as an implication of the form $\mathrm{X} \Rightarrow \mathrm{Y}$ where $\mathrm{X}, \mathrm{Y} \subseteq \mathrm{I}$ and $\mathrm{X} \cap \mathrm{Y}=\boldsymbol{\phi}$.

The sets of items (for short itemsets) $\mathrm{X}$ and $\mathrm{Y}$ is called antecedent (Left-Hand-Side or LHS) and consequent (Right-Hand-Side or RHS) of the rule.

$\{\mathrm{i} 1, \mathrm{i} 2\} \Rightarrow\{\mathrm{i} 3\}$ means, if $\mathrm{i} 1$ and $\mathrm{i} 2$ are bought customers also buy i3.

The best-known constraints are minimum thresholds on support and confidence.

Support $(\operatorname{Supp}(X))$ of an itemset $X$ - the proportion of transactions in the data set which contain the itemset.

$\operatorname{Supp}(\mathrm{X})=$ Number of transactions in which $\mathrm{X}$ occurred / Total number of transactions.

Confidence of a rule -

$\operatorname{Conf}(X=>Y)=\operatorname{supp}(X U Y) / \operatorname{supp}(X)$.

The goal of association rule mining is to discover all rules that have support and confidence greater than some userdefined minimum support and minimum confidence thresholds, respectively. 


\subsection{Frequent Itemsets - Structural Properties and Basic Discovery Approaches}

\subsubsection{Maximum Frequent Set}

Among all the frequent itemsets, some will be maximal frequent itemsets; they have no proper supersets that are themselves frequent. The maximum frequent set (or MFS) is the set of all the maximal frequent itemsets. The problem of discovering the frequent set can be reduced to the problem of discovering the MFS

\subsubsection{Closure Properties}

The set of all itemsets is partitioned, perhaps implicitly, into three sets:

1. Frequent: This is the set of those itemsets that have been discovered so far as frequent

2. Infrequent: This is the set of those itemsets that have been discovered so far as infrequent.

3. Unclassified: This is the set of all the other itemsets.

Initially, the frequent and the infrequent sets are empty. Throughout the execution, they grow at the expense of the unclassified set. The execution terminates when the unclassified set becomes empty, and then, of course all the maximal frequent itemsets are discovered.

Two closure properties can be used to immediately classify some of the unclassified itemsets

Property 1: If an itemset is infrequent, all it supersets must be infrequent, and they need not be examined further.

Property 2: If an itemset is frequent, all its subsets must be frequent, and they need not be examined further.

\subsubsection{Discovering Frequent Itemsets}

In general, it is possible to search for the maximal frequent itemsets either bottom-up or top-down. If all maximal frequent itemsets are expected to be short (close to 1 in size), it seems efficient to search for them bottom-up, uses only Property 1 to reduce the number of candidates. If all maximal frequent itemsets are expected to be long (close to $\mathrm{n}$ in size) it seems efficient to search for them top-down, uses only Property 2 to reduce the number of candidates as shown in Fig.1.

Bottom-up approach: It consists of repeatedly applying a pass, itself consisting of two steps. At the end of pass k all frequent itemsets of size $\mathrm{k}$ or less have been discovered. As the first step of pass $k+1$, itemsets of size $k+1$ each having two frequent $\mathrm{k}$-subsets with the same first $\mathrm{k}-1$ items are generated. Itemsets that are supersets of infrequent itemsets are pruned (and discarded), as of course they are infrequent (by Property 1). The remaining itemsets form the set of candidates for this pass. As the second step, the support of the candidates is computed (by reading the database), and they are classified as either frequent or infrequent.

Top-down approach: Starts with the single n-itemset and decreases the size of the candidates by one in every pass. When a k-itemset is determined to be infrequent, all of its (k-1)-subsets will be examined in the next pass. However, if a k-itemset is frequent, then all of its subsets must be frequent and need not be examined.

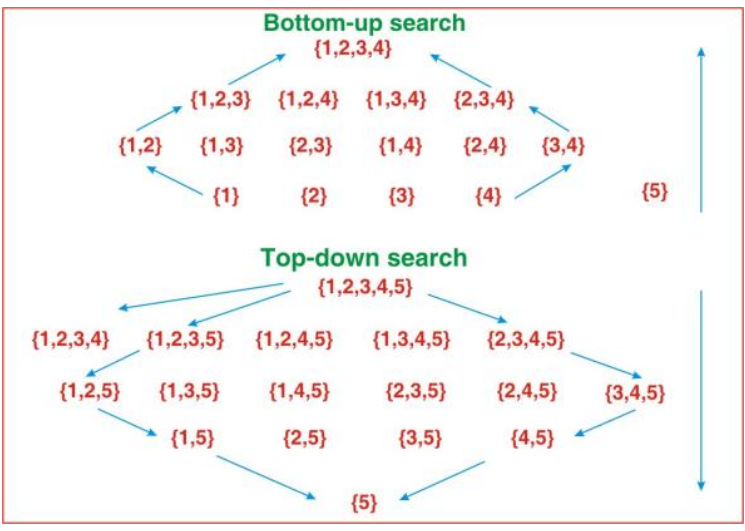

Fig. 1: Top-Down and Bottom-Up Searches

\section{ASSOCIATION RULE MINING ALGORITHMS}

\subsection{Apriori Algorithm}

The Apriori algorithm is a typical bottom-up approach algorithm. The Apriori algorithm repeatedly uses Apriorigen algorithm to generate candidates and then count their supports by reading the entire database once. Apriori-gen relies on Property 1.

\subsubsection{Join and Prune Procedures}

The candidate generation algorithm consists of a join procedure and a prune procedure. The join procedure combines two frequent k-itemsets, which have the same (k-1) prefix, to generate a $(\mathrm{k}+1)$-itemset as a new preliminary candidate. Following the join procedure, the prune procedure is used to remove from the preliminary candidate set all itemsets $\mathrm{C}$ such that some $\mathrm{k}$-subset of $\mathrm{C}$ is not a frequent itemset.

The join procedure of the Apriori-gen algorithm

Input: $\mathrm{L}_{\mathrm{k}}$, the set containing frequent itemsets found in pass $\mathrm{k}$

Output: Preliminary candidate set $\mathrm{C}_{\mathrm{k}+1}$

The prune procedure of the Apriori-gen algorithm

Input: Preliminary candidate set $\mathrm{C}_{\mathrm{k}+1}$ generated from the join procedure

Output: Final candidate set $\mathrm{C}_{\mathrm{k}+1}$, which does not contain any infrequent subset

\subsubsection{Reducing the Number of Candidates and the Number of Passes}

To design an algorithm that can efficiently discover both long and short maximal frequent itemsets, one might think of simply running both bottom-up and top-down programs at the same time. A key component of the approach is the use of information gathered in the search in one direction to prune more candidates during the search in the other direction. If some maximal frequent itemset is found in the top-down direction, then this itemset can be used to eliminate (possibly many) candidates in the bottom-up direction. The subsets of this frequent itemset can be pruned because they are frequent (Property 2 ). Of course 
if an infrequent itemset is found in the bottom-up direction, then it can be used to eliminate some candidates in the top-down direction (Property 1). This "Two-Way Search Approach" shown in Fig.2 can fully make use of

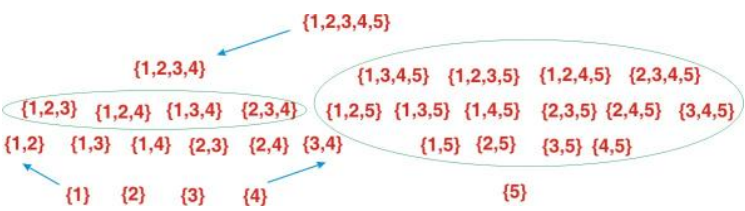

Fig. 2: Two-Way Search Approach

both properties and thus speed up the search for the maximum frequent set. Reducing the number of candidates is of critical importance for the efficiency of the frequent set discovery process, since the cost of the entire process comes from reading the database (I/O time) to generate the supports of candidates ( CPU time) and the generation of new candidates (CPU time)

\subsection{The Basic Pincer-Search Algorithm}

The Pincer-Search Algorithm relies on the combined approach for determining the maximum frequent.

\subsubsection{Pincer-Search Procedure}

Input: A database and a user-defined minimum support

Output: MFS, which contains all maximal frequent itemsets

The MFCS is initialized to contain one itemset, which consists of all the database items. The MFCS is updated whenever new infrequent itemsets are found. If an itemset in the MFCS is found to be frequent, then its subsets will not participate in the subsequent support counting and candidate set generation steps.

Pincer-Search reduces both the number of candidates and the number of passes.

\subsubsection{Two-Way Search by Using the MFCS}

The two-way search algorithm for discovering the maximum frequent set relies on a new data structure during its execution, the maximum frequent candidate set (MFCS).

Consider some point during the execution of an algorithm for finding the MFS

Let FREQUENT be the set of the itemsets known to be frequent.

Let INFREQUENT be the set of the itemsets known to be infrequent.

Then the maximum frequent candidate set (MFCS) is the minimum cardinality set of items satisfying the conditions.

$$
\begin{aligned}
& \text { FREQUENT } U\left\{2^{\mathrm{x}} / \mathrm{x} \in \text { MFCS }\right\} \\
& \text { INFREQUENT } \cap\left\{2^{\mathrm{x}} / \mathrm{x} \in \text { MFCS }\right\}=\phi
\end{aligned}
$$

MFCS is a superset of the MFS. When the algorithm terminates, the MFCS and the MFS are equal. The computation of the algorithm follows the bottom-up breadth-first search approach.
In each pass, in addition to counting supports of the candidates in the bottom-up direction, the algorithm also counts supports of the itemsets in the MFCS; this set is adapted for the top-down search. This will help in pruning candidates, but will also require changes in candidate generation. The subsets of the MFCS must not contain this infrequent itemset.

By using the MFCS some maximal frequent itemsets may be discovered in early passes. This early discovery of the maximal frequent itemsets can reduce the number of candidates and the passes of reading the database, which in turn can reduce the CPU time and I/O time. This is especially significant when the maximal frequent itemsets discovered in the early passes are long.

\subsubsection{Updating the MFCS}

Replace every such itemset (say X) by Y itemsets, each obtained by removing from $\mathrm{X}$ a single item (element) of $\mathrm{Y}$.

\subsubsection{Procedure of MFCS-gen}

Input: Old MFCS and the infrequent set $\mathrm{S}_{\mathrm{k}}$ found in pass $\mathrm{k}$ Output: New MFCS

\subsubsection{Prune procedure}

Input: Current MFCS and $\mathrm{C}_{\mathrm{k}+1}$ after join and recovery procedures

Output: Final candidate set $\mathrm{C}_{\mathrm{k}+1}$

\subsubsection{Candidate generation procedure}

Input: $\mathrm{L}_{\mathrm{k}}$, current MFCS, and current MFS

Output: New candidate set $\mathrm{C}_{\mathrm{k}+1}$

\subsection{The Dynamic Itemset Counting Algorithm}

1. Mark the empty itemset with a solid square Mark all the 1-itemsets with dashed circles. Leave all other itemsets unmarked.

2. While any dashed itemsets remain:

- Read M transactions (when the end of the transaction file is reached, continue from the beginning). For each transaction, increment the respective counters for the itemsets that appear in the transaction and are marked with dashes.

- If a dashed circle's count exceeds minsupp, turn it into a dashed square. If any immediate superset of it has all of its subsets as solid or dashed squares, add a new counter for it and make it a dashed circle.

- Once a dashed itemset has been counted through all the transactions, make it solid and stop counting it.

- $\quad$ Solid Square: $\square$ confirmed frequent itemset - an itemset that has finished 
counting and exceeds the support threshold minsupp.

- Solid circle:

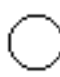

confirmed infrequent itemset - that has finished counting and it is below minsupp.

- Dashed square: : frequent itemset - an itemset which is still in counting that exceeds minsupp.

- Dashed circle: i.j $^{-j}$ suspected infrequent itemset - an itemset which is still in counting that is below minsupp.

Operations:

1. Add new itemsets

2. Maintain a counter for every itemset

3. Manage itemset states from dashed to solid and from circle to square. When itemsets become large, determine which new itemsets should be added because they could potentially be large.

\section{PERFORMANCE EVALUATION}

Assume support to be equal to $20 \%$. Since the number of transactions is 15 , it means that an itemset is supported by at least three transactions is a frequent set. Generate candidate itemsets and frequent itemsets, when minimum support count is $>2$.

Table 1: Sample Database

\begin{tabular}{|l|l|l|l|l|l|l|}
\hline & 1 & 2 & 3 & 4 & 5 & 6 \\
\hline 1 & 1 & 0 & 0 & 0 & 1 & 1 \\
\hline 2 & 0 & 1 & 0 & 1 & 0 & 0 \\
\hline 3 & 0 & 0 & 0 & 1 & 1 & 0 \\
\hline 4 & 0 & 1 & 1 & 0 & 0 & 0 \\
\hline 5 & 0 & 0 & 0 & 0 & 1 & 1 \\
\hline 6 & 0 & 1 & 1 & 1 & 0 & 0 \\
\hline
\end{tabular}

\section{Apriori Algorithm}

In a $9 \times 15$, in pass -3 only one set is present. This is MFS. So the algorithm stops.

\section{Pincer-Search Algorithm}

Here also in pass -3 only one set is present which is MFS so the algorithm stops.

Dynamic Itemset Counting Algorithm

This algorithm requires only 2.75 database passes instead of 3 passes.

For the association mining algorithms to be effective, the top-down search needs to reach the maximal frequent itemsets faster than the bottom-up search.

\section{TIME and SPACE COMPLEXITY COMPARISON}

Since transactions of itemsets are divided into groups by interval value, the work done by DIC algorithm [10] is increased. So complexity also increases. But the time taken to complete the algorithm is reduced.

In PINCER SEARCH algorithm, top-down and bottom-up approaches are applied on each step to find frequent itemset implicitly. So the amount of work done is increased in this case.

If the amount of transactions and itemsets are increased, then considerably time taken to complete the process will be increased. The program size of Apriori algorithm is 923 bytes and for Dynamic Itemset Counting Algorithm 1879 bytes.

Table 2: Run Time values

\begin{tabular}{|l|c|c|c|c|c|c|}
\hline \multirow{2}{*}{$\begin{array}{l}\text { DATABASE SIZE } \\
\text { ITEMSET X } \\
\text { TRANSACTIONS) }\end{array}$} & \multicolumn{3}{|c|}{ APRIORI } & \multicolumn{3}{c|}{ DIC } \\
\cline { 2 - 7 } & Start End (in Seconds) & \multicolumn{3}{c|}{ TIME (in Seconds ) } \\
\hline $4 \times 4$ & 51 & 55 & 4 & 8 & 10 & 2 \\
\hline $6 \times 6$ & 40 & 47 & 7 & 50 & 53 & 3 \\
\hline $6 \times 7$ & 50 & 57 & 7 & 34 & 37 & 3 \\
\hline $9 \times 10$ & 53 & 65 & 12 & 36 & 39 & 3 \\
\hline $9 \times 15$ & 39 & 55 & 16 & 44 & 48 & 4 \\
\hline
\end{tabular}

diagrammatically with the help of charts.
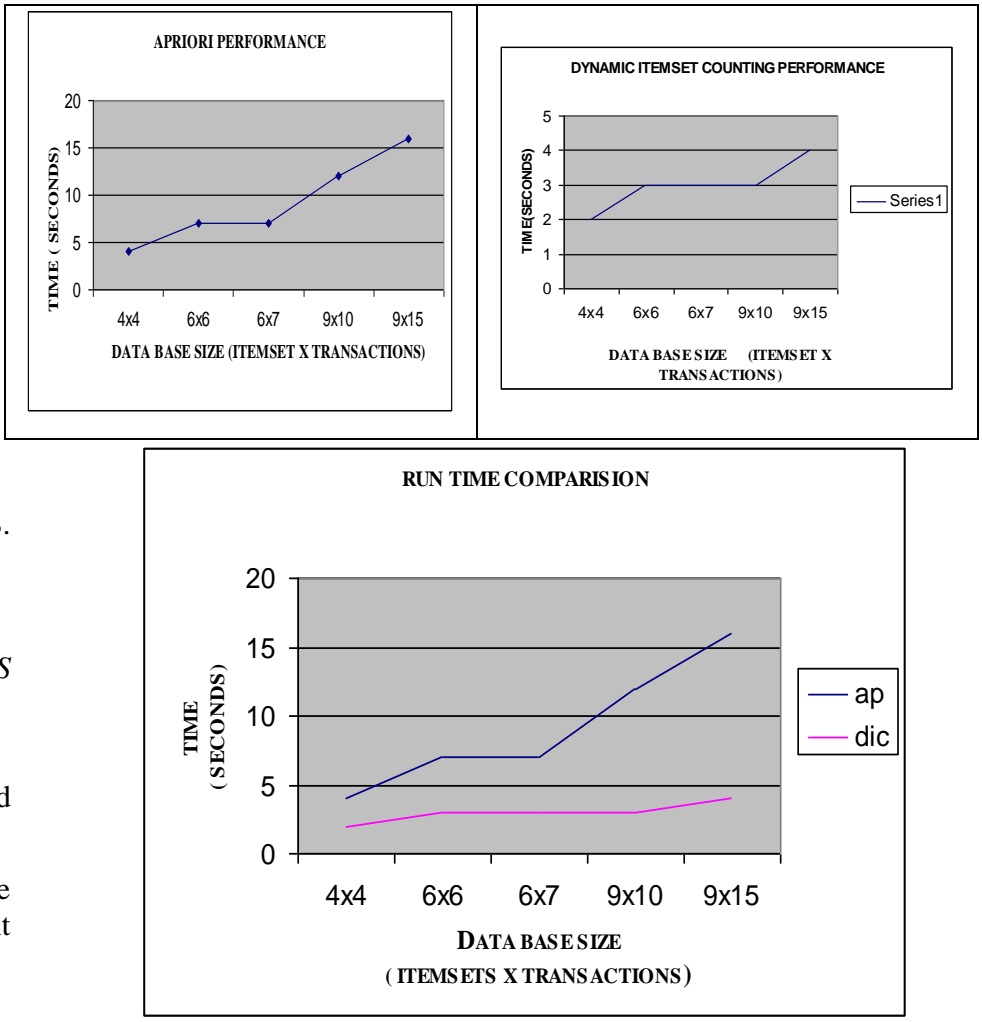

Fig. 3: Runtime and Space Complexity Comparison 


\section{CONCLUSION}

The maximum frequent set provides a unique representation of all the frequent set, and once it is known, all the required frequent subsets can be easily generated.

The Dynamic Itemset Counting algorithm could reduce both the number of times the database is read and the number of candidates.

Experiments show that the improvement of using this approach can be very significant, especially when some maximal frequent itemsets are long.

\section{FUTURE ENHANCEMENTS}

The performance of the Dynamic Itemset Counting algorithm in applications of discovering price changing patterns in stock market can be studied. It is worthwhile to study the performance of the Dynamic Itemset Counting Algorithm on problems having long maximal frequent itemsets. If the maximal frequent itemsets are distributed in a scattered manner, then the problem of discovering the MFS can be very hard. In this case, even Dynamic Itemset Counting Algorithm might not be able to solve it efficiently. Parallelizing the Dynamic Itemset Counting algorithm might be a possible way to solve this hard problem. The candidate set is divided in such a way that all the candidates that are subsets of an itemset in the MFCS are assigned to a same processor. Each processor can run totally independent. The issues to study would be the way to minimize the duplicate calculations and to maximize the use of available processors.

\section{REFERENCES}

[1] www.dama-ncr.org

[2] R. Agrawal, T. Imilienski, and A. Swami. Database Mining: A Performance Perspective. IEEE Transactions on Knowledge and Data Engineering, 5(6):914--925, December 1993.

[3] R. Agrawal, T. Imilienski, and A. Swami. Mining Association Rules between Sets of Items in Large Databases. Proc. of the ACM SIGMOD Int'l Conf. on Management of Data, pages 207--216, May 1993.

[4] R. Agrawal and R. Srikant. Fast algorithms for mining association rules. In Proceedings of the 20th VLDB Conference, Santiago, Chile, 1994.
[5] R. Agrawal and R. Srikant. Mining sequential patterns. In Proceedings of the 11th International Conference on Data Engineering, Taipei, Taiwan, 1995.

[6] R. Agrawal, K. Lin, S. Sawhney, and K. Shim. Fast similarity search in the presence of noise, scaling and translation in time-series databases. In Proc. of the Int'l Conf. on Very Large Data Bases (VLDB), 1995.

[7] R. Srikant and R. Agrawal. Mining generalized association rules. 1995

[8] M. Mehta, R. Agrawal, and J. Rissanen. Sliq: A fast scalable classifier for data mining. March 1996.

[9] H. Toivonen. Sampling large databases for association rules. Proc. of the Int'l Conf. on Very Large Data Bases (VLDB), 1996.

[10] Sergey Brin, Rajeev Motwani y Jeffrey D. Ullman z, Dynamic Itemset Counting and Implication Rules for Market Basket Data, Department of Computer Science, Stanford University, Shalom Tsur, R\&D Division, Hitachi America Ltd.

\section{AUTHOR'S PROFILE}

K. Fathima Bibi received her Bachelor's degree in Computer Science, Master's degree in Computer Science, and M.Phil. degree in Computer Science all from Bharathidasan University, Tiruchirappalli, Tamil Nadu, India. She cleared SET in October 2012. Presently she is pursing Ph.D. in the area of Data Mining at Bharathiyar University, Coimbatore, Tamil Nadu, India. At present, she is working as an Assistant Professor in the Department of Computer Science, Jamal Mohamed College (Autonomous), Tiruchirappalli, Tamil Nadu, India.

M. Nazreen Banu received her Bachelor's degree in Chemistry and Master's degree in Computer Applications, both from Bharathidasan University, Tiruchirappalli, Tamil Nadu, India. She gained her $\mathrm{PhD}$ degree from Nagoya Institute of Technology, Nagoya, Japan. She was a recipient of Japanese (MEXT) scholarship, from 2008 2012. She worked as an Assistant Professor at Jamal Mohamed College, Tiruchirappalli from 1999 - 2008. At present, she is working as a Professor in the Department of Computer Science \& Engineering, M.A.M College of Engineering, Tiruchirappalli. Tamil Nadu, India. Her research interests include Distributed \& Parallel processing, operating systems and Advanced computer networks 